\title{
P I 9-20. Allogeneic stimulation of the anti-viral APOBEC3G in human CD4+ T cells and prevention of SHIV infectivity in macaques immunized with HLA antigens
}

\author{
Y Wang*1, T Whittall ${ }^{1}$, J Scholler ${ }^{2}$, R Wyatt ${ }^{3}$, M Singh ${ }^{4}$, LA Bergmeier ${ }^{1}$, \\ E Bunnik ${ }^{5}$, H Schuitemaker ${ }^{5}$, O Shaw ${ }^{1}$, R Vaughan1, J Pido-Lopez ${ }^{1}$, T Seidl1, \\ K Babaahmady ${ }^{1}$, G Yang ${ }^{6}$, R Thorstensson7, G Biberfeld ${ }^{7}$ and T Lehner ${ }^{1}$
}

Address: ${ }^{1}$ Mucosal Immunology Unit, Kings College London, London, UK, ${ }^{2}$ Immunodex, Copenhagen, Denmark, ${ }^{3}$ National Institute of Health, Bethesda, MD, USA, ${ }^{4}$ Lionex Diagnostics \& Therapeutics, Braunschweig, Germany, ${ }^{5}$ Academic Medical Center, Amsterdam, Netherlands, ${ }^{6} \mathrm{Chinese}$ Centre for Disease Control and Prevention, Beijing, PR China and ${ }^{7}$ Swedish Institute for Infectious Disease Control, Stockholm, Sweden

* Corresponding author

from AIDS Vaccine 2009

Paris, France. 19-22 October 2009

Published: 22 October 2009

Retrovirology 2009, 6(Suppl 3):P340 doi:10.1 186/1742-4690-6-S3-P340

This abstract is available from: http://www.retrovirology.com/content/6/S3/P340

(c) 2009 Wang et al; licensee BioMed Central Ltd.

\section{Background}

APOBEC3G (A3G) is an intracellular anti-viral factor which deaminates cytidine to uridine. The activity of A3G is countered by Vif, which protects the virus by preventing incorporation of A3G into virions. A3G can be upregulated in vitro and in vivo to overcome Vif activity and inhibit HIV-1 or SIV infection.

\section{Methods}

Human CD4+ T cells were separated from PBMC of normal HIV-1- subjects and allostimulated by unmatched irradiated PBMC. A3G was assayed before and after allostimulation by RT-PCR, Western blots and immunofluorescence with $\mathrm{A} 3 \mathrm{G}$-specific antibodies. A3G expression in the subsets of memory CD4+ T cells was determined by immunofluorescence with antibodies to A3G, CD45RA and CCR7. Allo-immunization with recombinant HLAclass I and class II dextramers, HIVgp140, SIVp27 and the co-adjuvants HSP70 and Titermax (SC x4) was carried out in rhesus monkeys and they were challenged with SHIVSF162.P4.

\section{Results}

Allogeneic stimulation of human CD4+ T cells in vitro upregulated A3G mRNA ( $p=0.01)$. The mechanism of upregulation of A3G mRNA involves interaction between HLA on DC and TCR of CD4+ T cells, which is ZAP70 phosphokinase signalling dependent and induces CD40L and A3G mRNA expression in CD4+ T cells $(\mathrm{p}=0.001)$. In vivo significant inhibition in viral load or preventing infection was found against the heterologous viral challenge, when compared with unimmunized control animals. A significant increase in A3G mRNA was found already after the 1 st immunization ( $\mathrm{p}<0.02)$, with upregulation of CD4+CD95+CCR7+ central memory T cells.

\section{Conclusion}

In vitro allo-stimulation of human $\mathrm{CD} 4+\mathrm{T}$ cells and in vivo immunization with recombinant HLA-class I and II dextramers, trimeric HIVgp140, SIVp27, HSP70 and Titermax elicited significant upregulation of A3G in CD4+ memory $\mathrm{T}$ cells. A significant inverse correlation between the cumulative viral load and A3G in the central memory T cells suggests that A3G may have contributed to the prevention of SHIV SF162.P4 infection. 